Environmental factors

We identified and implemented a number of change ideas, using Plan-Do-Study- Act methodology, regularly meeting to review progress and plotting our data on a run chart.

Key patient interventions included a "Mutual Respect" exercise and regular "Community Meetings".

Staff interventions included use of Safety Crosses, Daily Safety Briefings and the Broset Violence Checklist (BVC).

Environmental factors were continually assessed and escalated as appropriate.

We raised awareness of our project and gained feedback by creating a dedicated notice board, providing a staff information session and including it as an agenda item at ward meetings.

Our project measures were identified as:

Outcome: Number of level 1 violent incidents occurring per week

Balancing: Number of incidents in other categories; Patient satisfaction

Process: Staff safety rating; Engagement with interventions

Result. Unfortunately, we were unable to meet our initial goal and there continued to be considerable variation in the number of weekly incidents.

We believe this was attributable to several factors, including the level of acuity within the ward during the project timeframe. It was noted that a relatively small number of patients contributed to a large proportion of the total incidents. Our results, therefore, did not reflect the success of interventions with other patients on the ward.

Despite this, we noted improvements in terms of patient and staff engagement with the project, including subjective reports of staff safety during shifts.

Conclusion. The unpredictable and complex nature of the PICU setting cannot be under-estimated and this ultimately impacted on achieving our intended outcome.

We do feel, however, that the project has had a positive impact and we hope we can build on this progress over the coming months.

Further interventions are being explored, including personalised daily activity schedules and attempts to reduce levels of continuous observations.

A quality improvement project on nicotine replacement therapy in Shannon Clinic (Northern Ireland)

Eileen Moss

Shannon Clinic

doi: 10.1192/bjo.2021.564

Aims. The aims of this quality improvement project were to determine if Nicotine Replacement Therapy was being prescribed correctly in Shannon Clinic in Northern Ireland and also to improve the rates of correct prescribing of Nicotine Replacement Therapy in the aforementioned unit.

Background. There are several different types of Nicotine Replacement Therapy currently available. Shannon Clinic is a smoke-free clinical environment therefore patients who smoke are offered Nicotine Replacement Therapy on admission. When I was working at Shannon Clinic I became aware that there was no clear guidance available to medical staff on the wards regarding prescribing Nicotine Replacement Therapy and therefore I decided to carry out this quality improvement project.

Method. An audit of drug charts was done on the patients who were under the care of the consultant that I worked with. In total nine drug charts were included in the audit. After the audit was complete, I produced a poster to show how to correctly prescribe Nicotine Replacement Therapy. A copy of this poster was placed on each ward in Shannon Clinic. After a period of time the drug charts were re-audited to see if there had been an improvement in the rates of correct prescribing of Nicotine Replacement Therapy.

Result. In total, $22 \%$ of the drug charts which were included in the audit had Nicotine Replacement Therapy prescribed incorrectly on them. After the inclusion of a poster outlining how to prescribe Nicotine correctly on each ward in Shannon Clinic, $0 \%$ of drug charts had Nicotine Replacement Therapy prescribed incorrectly on them. This was an improvement of $22 \%$. Conclusion. This quality improvement project was successful at reducing the rates of incorrect Nicotine Replacement Therapy in Shannon Clinic. In the future it is my hope that this quality improvement project should lead to the correct prescribing of Nicotine Replacement Therapy for all patients in Shannon Clinic. It should also lead to an increased awareness regarding the different types of Nicotine Replacement Therapy for medical staff working in this clinical unit.

\section{A study to improve the quality of writing clinic letters to patients attending the outpatient clinic \\ Irangani Mudiyanselage ${ }^{1 \star}$ and Madhvi Belgamwar ${ }^{2}$ \\ ${ }^{1}$ Derbyshire Health Care NHS Foundation Trust and ${ }^{2}$ Consultant \\ Psychiatrist, Derbyshire Healthcare NHS Foundation Trust \\ ${ }^{\star}$ Corresponding author.}

doi: 10.1192/bjo.2021.565

Aims. In many countries (including the UK and Australia) it is still common practice for hospital doctors to write letters to patients' general practitioners (GPs) following outpatient consultations, and for patients to receive copies of these letters. However, experience suggests that hospital doctors who have changed their practice to include writing letters directly to patients have more patient centred consultations and experience smoother handovers with other members of their multidisciplinary teams. (Rayner et al, BMJ 2020)

The aim of the study was to obtain patient's views to improve the quality of clinical letters sent to them, hence the level of communication and standards of care.

Method. An anonymous questionnaire was designed and posted to collect information from patients attending one of the South County Mental Health outpatient clinic in Derbyshire. 50 random patients were selected between March to November 2020. Patients were asked to provide suggestions to improve the quality of their clinic letters written directly to them and copies sent to their GPs. Result. Out of 50 patients $48 \%(\mathrm{n}=24)$ responded. Majority of patients (92\%) expressed their wish to receive their clinic letters written directly to them and $79 \%$ preferred to be addressed as a second person in the letters. More than half $(54 \%, \mathrm{~N}=13)$ of them would like to have letter by post. Majority of them $(92 \%$, $\mathrm{N}=22$ ) wished to have their letter within a week of their consultations.

Patients attending clinics felt that the communication could be better improved through writing clearly: a) reflection of what was discussed during the consultation b) updated diagnosis c) a clear follow-up plan d) current level of support e) medication change f) emergency contact numbers g) actions to be carried out by their GP and further referrals should there be any.

Conclusion. Patients in community prefer to have their clinic letters directly addressing them in second person. It was noted that the letters needed to reflect accurately on what was discussed during the consultation in order to have patient centered consultations. This in turn would improve communication and thus rapport, trust and overall therapeutic relationship. 